\title{
Efficacy of an enhanced linkage to HIV care intervention at improving linkage to HIV care and achieving viral suppression following home-based HIV testing in rural Uganda: study protocol for the Ekkubo/ PATH cluster randomized controlled trial
}

Susan M. Kiene ${ }^{1 *}$, Seth C. Kalichman², Katelyn M. Sileo ${ }^{3}$, Nicolas A. Menzies ${ }^{4}$, Rose Naigino ${ }^{5}$, Chii-Dean Lin ${ }^{6}$, Moses H. Bateganya ${ }^{7}$, Haruna Lule ${ }^{8}$ and Rhoda K. Wanyenze ${ }^{5}$

\begin{abstract}
Background: Though home-based human immunodeficiency virus (HIV) counseling and testing (HBHCT) is implemented in many sub-Saharan African countries as part of their HIV programs, linkage to HIV care remains a challenge. The purpose of this study is to test an intervention to enhance linkage to HIV care and improve HIV viral suppression among individuals testing HIV positive during HBHCT in rural Uganda.

Methods: The PATH (Providing Access To HIV Care)/Ekkubo Study is a cluster-randomized controlled trial which compares the efficacy of an enhanced linkage to HIV care intervention vs. standard-of-care (paper-based referrals) at achieving individual and population-level HIV viral suppression, and intermediate outcomes of linkage to care, receipt of opportunistic infection prophylaxis, and antiretroviral therapy initiation following HBHCT. Approximately 600 men and women aged 18-59 who test HIV positive during district-wide HBHCT in rural Uganda will be enrolled in this study. Villages (clusters) are pair matched by population size and then randomly assigned to the intervention or standard-of-care arm. Study teams visit households and participants complete a baseline questionnaire, receive HIV counseling and testing, and have blood drawn for HIV viral load and CD4 testing. At baseline, standard-of-care arm participants receive referrals to HIV care including a paper-based referral and then receive their CD4 results via home visit 2 weeks later. Intervention arm participants receive an intervention counseling session at baseline, up to three follow-up counseling sessions at home, and a booster session at the HIV clinic if they present for care. These sessions each last approximately $30 \mathrm{~min}$ and consist of counseling to help clients: identify and reduce barriers to HIV care engagement, disclose their HIV status, identify a treatment supporter, and overcome HIV-related stigma through links to social support resources in the community. Participants in both arms complete interviewer-administered questionnaires at six and 12 months follow-up, HIV viral load and CD4 testing at 12 months follow-up, and allow access to their medical records.

(Continued on next page)
\end{abstract}

\footnotetext{
* Correspondence: skiene@mail.sdsu.edu

'Division of Epidemiology and Biostatistics, Graduate School of Public Health,

San Diego State University, 5500 Campanile Drive (MC-4162), San Diego, CA

92182, USA

Full list of author information is available at the end of the article
} 
(Continued from previous page)

Discussion: The findings of this study can inform the integration of a potentially cost-effective approach to improving rates of linkage to care and HIV viral suppression in HBHCT. If effective, this intervention can improve treatment outcomes, reduce mortality, and through its effect on individual and population-level HIV viral load, and decrease HIV incidence.

Trial registration: NCT02545673

Keywords: Home-based HIV counseling and testing (HBHCT), Linkage to care, HIV viral suppression, Health behavior intervention, Cluster-randomized controlled trial, Uganda

\section{Background}

In the fight against human immunodeficiency virus (HIV), efforts to expand access to antiretroviral treatment (ART) are being prioritized globally. ART has the ability not only to improve health outcomes for the individual, but also in the prevention of ongoing HIV transmission through reducing viral load (VL). The push towards universal access to ART is reflected in the United Nations Joint Programme on HIV/Acquired Immunodeficiency Syndrome (AIDS) "90-90-90" goals, which aim to get $90 \%$ of people living with HIV (PLHIV) aware of their status, $90 \%$ of those diagnosed linked to care and receiving HIV treatment, and $90 \%$ of those receiving treatment virally suppressed by 2020 [1]. The 2016 World Health Organization (WHO) consolidated guidelines recommend all PLHIV be started on ART immediately upon diagnosis, regardless of CD4 cell count or disease stage [2]. However, significant challenges in getting PLHIV linked to HIV care and on ART remain, including in sub-Saharan Africa (SSA), the region most affected by the HIV epidemic. It is estimated that only $53 \%$ of those eligible for ART in SSA were receiving it in 2015 [3]. Several systematic reviews of studies in SSA demonstrate low rates of linkage to care after HIV testing [4-7], with up to two-thirds of patients lost to follow up before initiation of ART [7]. Thus, strategies to facilitate linkage into HIV care in SSA are needed, especially as the number of people eligible for ART grows as countries implement "test and treat" recommendations throughout the region.

In Uganda, it is estimated that only $65 \%$ of the 1.46 million PLHIV were aware of their status in 2015, of which only $51 \%$ were receiving ART [8]. Moreover, the number of people eligible for ART is expected to increase, as the revised 2016 Ugandan Ministry of Health $(\mathrm{MOH})$ national treatment guidelines, which take effect in 2017, promote expansion of ART to all PLHIV, and put forth a number of strategies to increase the number of people aware of their HIV status and on treatment [8]. Home-based HIV counseling and testing (HBHCT) is one such approach, where HIV counseling and testing teams travel door-to-door and offer HIV testing services. HBHCT is especially important in the era of expanded access to ART and "treatment as prevention," since HBHCT, compared to other modalities of testing, identifies HIV infections at an earlier disease stage and reaches more people who have never had an HIV test [9]. Moreover, HBHCT may be the most cost-effective testing strategy per person tested [9] and per new HIV infection identified [10]. However, though HBHCT is being widely implemented throughout SSA, [11] very few studies have examined linkage to care and treatment for individuals who test HIV positive at home [12-14].

The most common linkage-to-care approach with HBHCT, paper-based referral to care sometimes with follow-up home visits, has not been rigorously evaluated, and a number of HBHCT-specific barriers may limit its effectiveness. A systematic review on linkage to care in developing countries identified the following as the most commonly cited barriers: psychosocial factors (fear of being seen at the clinic, fear of disclosing, not feeling sick), structural and economic barriers (transportation costs, distance to the clinic), and health system factors (drug stock-out) [5]. Research evidence in Uganda has similarly documented these barriers [15], with pervasive HIV stigma, especially in rural areas, a particularly prominent barrier to care [16-19]. Such barriers may limit implementation of HBHCT; while HBHCT minimizes stigma and structural barriers of HIV testing (e.g., transportation) by bringing the test directly to patients [20], such barriers may remain an issue for linkage to care, as testing occurs away from the health facility and in a community setting, where fear of stigma may be elevated. Moreover, there is less structure in place for tracking patients testing positive in HBHCT, which is often done once without any follow-up [21]. In Uganda, HBHCT has been shown to be highly acceptable; $94 \%$ of individuals offered testing in a district-wide HBHCT study accepted testing and received their results [22]. However, only $10.5 \%$ of those who tested HIV positive were linked to ART [22]. In another study, only $58 \%$ of those who tested positive in HBHCT were linked to care within 12 months [23]. In HBHCT studies across SSA, linkage ranges from $42 \%$ to $97 \%$ within 1-12 months follow-up [24-30]. 
Low rates of linkage to HIV care during HBHCT highlights the need for interventions aimed to improve access to HIV care and treatment for individuals testing HIV positive in the home. The literature on HBHCT programs suggests that those with two follow-up home visits with counseling have better linkage compared to those with one or no follow-up home visits [24-30]. This finding is supported by recent trials in South Africa and Uganda, which found lay counselor facilitation and follow-up visits increased linkage [14, 31]. Based on evidence of the strong influence of HIV stigma, linkage to care interventions should be optimized in the context of rural $\mathrm{HBHCT}$ if they are to effectively reduce HIV stigma while addressing other barriers to care. A review of interventions to reduce HIV stigma found those providing social support to PLHIV were effective at reducing stigma [32], and emotional support through support groups has been shown to reduce internalized stigma among HIV-positive women in South Africa [33]. While no intervention studies have further linked reductions in stigma to subsequent improvements in linkage to care, a randomized trial in Uganda showed that counseling and social support via home visits doubled the likelihood of linkage to care for newly-diagnosed PLHIV [34]. Other data similarly suggests that social support may increase care linkage and retention [35-37], as well as reciprocal relationships between social support and HIV stigma [38]. Thus, providing PLHIV social support soon after diagnosis may protect them from internalizing HIV stigma and buffer the effects of enacted HIV stigma, which in turn may improve linkage to HIV care.

This paper describes the protocol for a clusterrandomized controlled trial (CRCT) to test an enhanced linkage to care intervention during $\mathrm{HBHCT}$ in rural Uganda. Our Providing Access To HIV care (Providing Access to HIV Care (PATH)/ Ekkubo) intervention enhances a similar strategy tested by Coates, Wanyenze, Bangsberg, and colleagues [39] that was found to reduce time to HIV care and treatment among patients receiving opt-out HIV testing in health facilities in Uganda. The intervention facilitated linkage by: providing orientation to the HIV care system, counseling to help clients identify and reduce barriers to engagement in care, and assistance disclosing and identifying a treatment supporter. The PATH/Ekkubo intervention described in this paper enhances the original intervention in order to increase its impact on multiple outcomes, including HIV viral suppression, and adapts the intervention to address barriers specific to HBHCT by providing additional counseling with increased emphasis on HIV stigma reduction through increased social support. We test this intervention in a CRCT, with randomization at the level of the village rather than at individual level, to avoid potential contamination since village residents interact with one another regularly in our study setting. The specific aims are:

1. Compare the efficacy of the enhanced linkage to care intervention (PATH) vs. standard-of-care (paper-based referrals) at achieving individual and population-level HIV viral suppression, and intermediate outcomes of linkage/time to care, time to/receipt of ART. We hypothesize that the PATH intervention study arm will have a higher proportion of participants who are: a) virally suppressed at 12-month follow up, and will have shorter time to: b) HIV care and c) ART initiation, among those eligible, than the standard-of-care study arm. We also hypothesize that the effect of the intervention on HIV viral suppression will be mediated by intermediate behavioral outcomes: linkage to care and ART initiation. Finally, at the cluster level, we hypothesize that villages randomized to the intervention arm will have higher rates of population-level HIV viral suppression than those in the standard-of-care study arm at 12-month follow-up.

2. Using the standard-of-care group as a natural history control, collect longitudinal data on barriers to and facilitators of linkage to and retention in care and treatment and HIV viral suppression.

3. Estimate the cost and cost-effectiveness of the intervention, as compared to standard-of-care HBHCT, in terms of: viral suppression, linkage to care, and receipt of ART.

\section{Design and methods}

\section{Setting}

The study is being conducted in the rural districts of Butambala, Mpigi, Mityana, and Gomba in central Uganda. Health services, including HIV care and treatment, are provided free at government health facilities. In line with WHO recommendations [40], the Ugandan $\mathrm{MOH} 2016$ guidelines, now expand access to lifelong ART to all adolescents and adults living with HIV immediately upon diagnosis regardless of CD4 count, in contrast to their previous eligibility threshold of CD $4<500$ [8]. Our study began before these new guidelines were nationally disseminated and implemented. Under the currently implemented $\mathrm{MOH}$ protocol, at the first clinic visit, a CD4 test is done and patients are started on cotrimoxazole. Once CD4 results are available ( $<2$ weeks), those eligible for ART receive pre-treatment counseling and are given an appointment to come back 1 week later to initiate ART. HIV care and treatment, as well as peer support groups for PLHIV, are available at all district hospitals and level III health centers. As the 2016 policy for the expansion of lifelong ART is rolled out during 
2017 all HIV-positive individuals will become eligible for ART regardless of their CD4 count. Throughout Uganda, a cadre of lay workers, constituting the Village Health Team (VHT), support community health-related activities and serve as liaisons between the community and the health facilities.

\section{Study design}

The trial design is a matched-pair two-arm, clusterrandomized design with clusters being villages. One arm is the PATH/Ekkubo intervention arm and the other is a standard-of-care (control) arm. Villages are randomized to study arms. We will include 40 villages with approximately 600 participants across all clusters (villages). We will conduct structured interviews at baseline (pre-intervention), and at six and 12-month follow-up. Similarly, blood draws for HIV viral load and CD4 testing will be done at baseline and at 12-month follow-up. The design is illustrated in the CONSORT Diagram, Fig. 1.

\section{Study population}

The study population is adults aged 18 to 59 as well as emancipated minors (which according to the Ugandan National Council for Science and Technology are those under 18 who are married, have children, or are pregnant), residing in villages selected for inclusion in the study. Individuals who have accepted HBHCT and provide written (or thumb-printed) informed consent will be eligible to participate in the study.

\section{Sampling and randomization}

The sampling scheme is stratified clustering with matched-pair sampling. Within each district we will pair match the 40 clusters (villages) by population size and proximity to major and regional roads, since the latter may facilitate access to health services because of greater availability of transportation. Using computer generated random numbers, we will randomly assign one village to be in the intervention arm and the other to the standard-of-care arm. Since village residents interact with one another regularly, we chose to randomize at the level of the village instead of randomizing individuals to study condition to avoid potential contamination. We will ensure that villages assigned to different study conditions are not within $4 \mathrm{~km}$ ( $1 \mathrm{~h}$. by foot) of each other.

\section{Procedures}

\section{Community mobilization for HBHCT}

Following the Ugandan $\mathrm{MOH}$ guidelines [41], this study provides village-wide $\mathrm{HBHCT}$ across the study villages. HBHCT and study recruitment is being conducted over approximately 46 months, beginning in November 2015 and ongoing through August 2019. The HBHCT teams will work in two villages at a time (one village from each study arm), and proceed to the next two villages upon completion, and so on. Prior to beginning recruitment in a village, members of our research team will meet with political, religious, and other village leaders, and identify VHT members to will serve as "mobilizers;" together VHTs and community leaders will inform the residents about HBHCT and the upcoming study. Using existing lists of households and maps of homesteads within the village, VHT mobilizers will visit all households in the village and seek permission for the HBHCT team to visit. For the households who agree to have the team visit, the VHT will set an appointment for this visit. The VHT will accompany the team to the household on the scheduled visit day, introduce the team and then leave to allow the recruitment, informed consent, data collection, and HIV testing to occur. The teams will set appointments to re-visit households in which household members were absent. The HBHCT teams consist of a study interviewer, a laboratory technician, and an HIV counselor who were all previously trained in general research procedures. Additionally they were trained in the study protocol and procedures.

\section{Eligibility, recruitment, and informed consent procedures HBHCT and brief baseline questionnaire}

After the team is introduced by the VHT during the HBHCT visit, the interviewer will verbally screen household members for eligibility for HIV testing and study participation using a brief questionnaire. Eligibility for the baseline interview includes being 18-59 years of age or an emancipated minor, accepting HIV testing, speaking Luganda-the predominant language spoken in the study area, or English and residing in the household. Individuals who don't normally sleep in the house will be excluded because they may live outside of the study area). We will obtain written informed consent for conducting the baseline interview and HIV testing. Those who consent will be interviewed before being tested for HIV as it would be insensitive to ask participants to answer interview questions after learning they are HIV positive. We expect approximately 25,000 individuals will participate in this aspect of the study.

\section{Intervention trial}

Individuals who participated in the brief baseline questionnaire interview and are identified as newly diagnosed HIV positive by our HBHCT team, as well as individuals who disclose as being known to be HIV positive during the baseline questionnaire and are confirmed positive in re-testing by the HBHCT team, but have never linked to HIV care, will be eligible for participation in the intervention trial. Only one person per household will be included in the intervention trial to ensure independence in the data. Using computerized random selection, we 


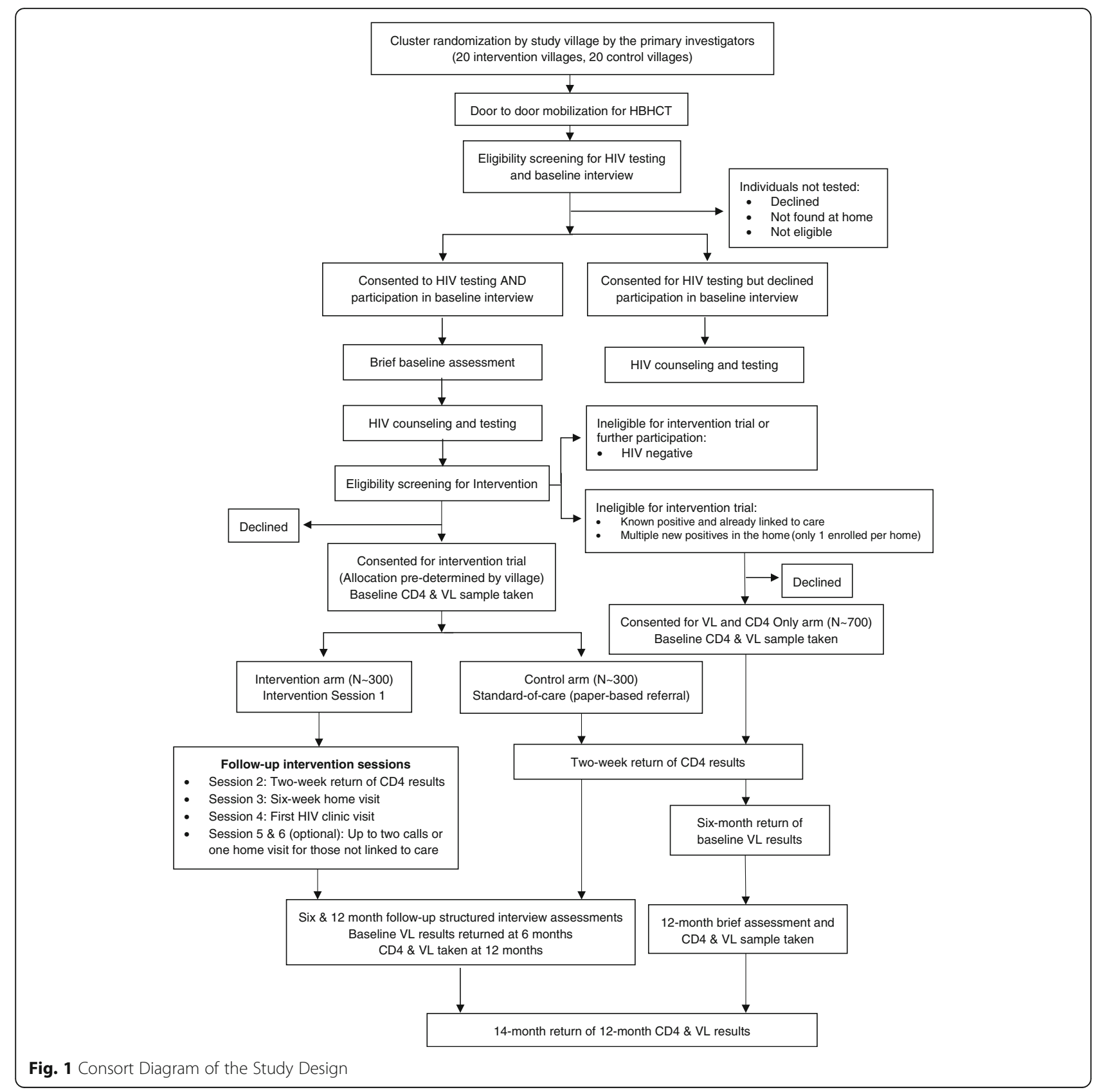

will randomly select one of the eligible HIV-positive household members for inclusion in the intervention trial. For ethical reasons the same follow-up support (intervention or control) will be offered to other newly diagnosed or HIV-positive household members not in care as will be provided to the household member included in the intervention study. A total of approximately 600 participants (approximately 300 in intervention and 300 in control arm villages) who are eligible and provide written informed consent to participate in the intervention trial will be enrolled.

\section{CD4 and HIV VL testing only}

We anticipate that approximately 700 participants who complete the baseline questionnaire and test HIV positive will be excluded from the intervention trial because they are already aware of their status and have linked to HIV care, or because there are multiple new HIV diagnoses within a household. These individuals will be offered participation in a "CD4 and VL testing only" group. Including this group will allow us to have HIV VL measures from all consenting new or known PLHIV in the included villages which provides us the ability to 
assess the population-level effects of the intervention. Study staff will obtain written informed consent for the collection of CD4, VL, and a brief questionnaire interview at 12-month follow-up. See Fig. 1 for a flowchart depicting the study procedures and sample size approximation per study arm.

\section{HIV testing procedures}

HBHCT will be conducted according to the $\mathrm{MOH}$ protocol for HBHCT [41] by study staff trained in HIV testing and counseling procedures. According to the protocol, counseling consists of pre-test counseling and giving results with post-test counseling. Pre-test counseling includes: reviewing the test process, discussing the understanding of potential test results, and assessing risk for HIV infection. Rapid tests will be used which provide results in approximately $15 \mathrm{~min}$. In the measures section we provide details on the HIV testing algorithm. When the results are ready, the counselor will provide the test results to the individual (or couple if applicable). The information provided will depend on whether the test is positive or negative. If the result is negative, the counselor will discuss HIV prevention (condom use, ways to reduce risk) and encourage future testing. If the result is positive the counselor will provide supportive counselling, discuss disclosure (if applicable), emphasize the importance of partner testing (if applicable), discuss HIV care and treatment available at the nearest health facilities and the laboratory technician will take a blood sample for CD4 and HIV VL testing. After the counselor tells the client his/her HIV test results, for those who are newly diagnosed, counselors will provide linkage to care following either the standard-of-care (paper-based referrals) or the PATH/Ekkubo intervention protocol, as detailed in the following sections. Control counselors will be trained to deliver standard-of-care linkage only, and will work only in villages allocated to the control arm. The intervention counselors will be trained in the PATH/Ekkubo intervention protocol over a four-day training and will work only in intervention villages.

\section{Description of the study arms \\ Control arm: Standard-of-care linkage (see Table 1)}

\section{During HBHCT}

In villages randomized to the standard-of-care arm, the control counselor will provide information on HIV care and treatment and provide the client with a referral to HIV care and a list of health facilities, including location and HIV clinic hours, in their area that provide free HIV care.

\section{Two week CD4 results home visit}

Two weeks after HBHCT, the control counselor will return to the participant's home and provide them with
Table 1 Linkage to care in the control arm: Standard-of-Care

\begin{tabular}{ll}
\hline Baseline HBHCT visit & Provide information on HIV care and \\
10 min & treatment available including HIV clinic \\
& locations and hours and provide \\
& referral to care \\
Two-Week CD4 results home & $\begin{array}{l}\text { Provide CD4 results and a paper-based } \\
\text { visit }\end{array}$ \\
15 min & referral to care. Explain logistics of how \\
& HIV care
\end{tabular}

Note: Control arm activities follow standard of care for linkage referrals in Uganda. HBHCT home-based HIV counseling and testing

their CD4 test results, and explain that they should take the $\mathrm{CD} 4$ results card with them when they present for HIV care. The counselor will also explain what the CD4 count means and if it indicates that they are eligible for ART, until the district adopts the $\mathrm{MOH}$ guidelines for immediate ART initiation, at which point they will be told they are eligible for ART regardless of CD4 test result. The two-week interval of return of CD4 results was the standard-of-care before "test and start" began to be adopted. With expansion of universal treatment, CD4 testing will no longer be a requirement for treatment initiation.

\section{PATH/Ekkubo intervention arm: Enhanced linkage to care and treatment (see Table 2)}

The intervention was originally developed and tested in a randomized trial with urban outpatients receiving optout HIV testing by Dr. Wanyenze [39]. Due to logistical differences between outpatient opt-out testing and HBHCT, we have made adjustments to include additional counselling sessions, to encourage and facilitate disclosure and seeking social support to address HIVrelated stigma to increase the intervention's effect on multiple outcomes-beyond those examined in the previous trial. The development of stigma-reduction elements of the intervention were guided by the HIV Stigma Framework [42].

The overall goal of the PATH/Ekkubo intervention is to identify and reduce barriers to HIV care through multiple follow-up counseling sessions following HBHCT. The intervention content includes up to six sessions delivered over a 10-week period. The core components and session schedule are described in Table 2. The first session begins after post-test HIV counseling during the initial HBHCT home visit and subsequent sessions are at 2 weeks and 6 weeks post HBHCT with optional sessions at 8 and 10 weeks for those who have not linked to care by 6 weeks; in addition there is a booster counseling session at the clinic when the client first presents to the HIV clinic. The components of the intervention include: linkage to care and referral, provision of CD4 results during the two-week visit, orientation to the HIV care system and the assessment of potential for adverse 
Table 2 Intervention arm: Enhanced Linkage to Care

Session 1, Baseline home
visit
Protocol Components 1-8
30 min

Intervention session core components

1. Explain linkage to HIV care

2. Assessment of potential adverse effects

3. Assess needs/barriers to HIV care

4. Provide counseling to overcome barriers

a. Assess HIV-related stigma

b. Counseling to overcome stigma

c. Identify sources of social support

d. Provide referrals for social support

e. Counseling to address other barriers

5. Help the client plan for disclosure,

seeking social support, and HIV stigma

a. Create a disclosure plan

b. Identify treatment supporter(s)

c. Encourage support seeking

6. Orient client to the HIV care system

and process

7. Elicit client's intention to seek HIV care

8. Set the time/date for two-week CD4

result home visit

Session 2, Two-week CD4

results home visit

30 min

1. Provide CD4 results and explain what they mean. If not eligible for treatment explain importance of repeat CD4 testing every 3 months

2. Review plans and discuss client's plans from Session 1

3. Assess if client has linked to care

4. Assess the participant's needs/barriers

to care and provide counseling/referrals

a. If patient has not been linked:

As appropriate, repeat components 1-7

from Session 1

b. If patient has been linked: As appropriate, repeat components 1-6 from Session 1 in relation to continued linkage

5. Assess HIV-related stigma and if client

has disclosed and sought social support.

6. As needed provide counseling on

seeking social support

7. Assess and discuss disclosure

8. Discuss treatment supporter

9. Review barriers and plans for overcoming

barriers and getting social support

10. Elicit client's intention to seek HIV

care/attend second clinic visit

11. Set the date/time for the next

home visit

Session 3, Six-week home visit Repeat steps outlined in Session 2

$30 \mathrm{~min}$

Session 4, First HIV clinic visit $30 \mathrm{~min}$

Optional Session 5 \& 6, Additional visit/phone calls $30 \mathrm{~min}$
1. Congratulate client on attending the HIV clinic

2. Assess client's barriers to attending a second clinic visit

3. Assess if client sought social support and if needed provide counseling on seeking social support

4. Address any other barriers to care and any medical needs

5. Review barriers to care, plans for overcoming barriers and getting social support

6. Elicit intention to attend the next clinic visit

Up to two calls those lost to follow up, repeat Session 3

If cannot be reached via phone, one home visit for those lost to follow up, repeat Session 3 effects related to HIV diagnosis and linkage to care (e.g., intimate partner violence). The counselor assesses needs and barriers to linkage (e.g., coping skills, social support, HIV-related stigma, and additional barriers), provides counseling and referrals to overcome identified barriers, and provides additional help planning for disclosure, seeking social support, and dealing with HIV-related stigma. Finally, the session ends with the elicitation of the client's intention to seek HIV care. Each subsequent session includes reassessment of linkage status and barriers to care and counseling to overcome barriers, building on the counseling provided in the previous sessions. If at any point during the intervention period the client has linked to HIV care, the counselor aims to reinforce engagement in care and assess/reduce barriers to continued engagement.

\section{Data collection procedures and measures}

Using a computerized structured questionnaire, a brief baseline interview will be conducted during the HBHCT visit for all participants consenting to HIV testing and interview. Following completion of the interview, the team will undertake HIV counseling and testing following the Ugandan $\mathrm{MOH}$ protocol for HBHCT [41]. If the result is positive and the participant has provided informed consent for enrollment in either the intervention trial or the CD4 and VL monitoring only group, the health worker will take blood for CD4 and HIV VL testing. Follow-up interviews will be conducted at six and 12-months followup for participants enrolled in the intervention trial, using a computer-based structured questionnaire $(<60 \mathrm{~min}$ duration). The data collection schedule, procedures, and instruments will be identical in both study arms and follow-up interviewers will be blinded to study arm assignment. A brief, 12-month questionnaire will be administered with participants in the CD4 and VL only group. For both intervention trial and CD4 and VL groups, we and will also take a blood sample for CD4 and HIV VL testing at 12 months, which will be returned to all participants during a 14-month home visit. Additionally, study staff will abstract data regarding attendance at the HIV clinic, CD4 count, and treatment regimen from medical records at each of the participating clinics as a secondary data source for some of the study outcomes. Cost data will be collected from activity logs and accounting records, and through micro-costing of intervention activities. Details of the study variables, measures, and timeframe for collection are described below and in Table 3.

- HIV status (baseline). Health workers will obtain a finger stick capillary blood sample to run the HIV rapid tests. Following WHO recommendations to assure HIV testing quality in high prevalence settings [43] and the Ugandan $\mathrm{MOH}$ recommended 


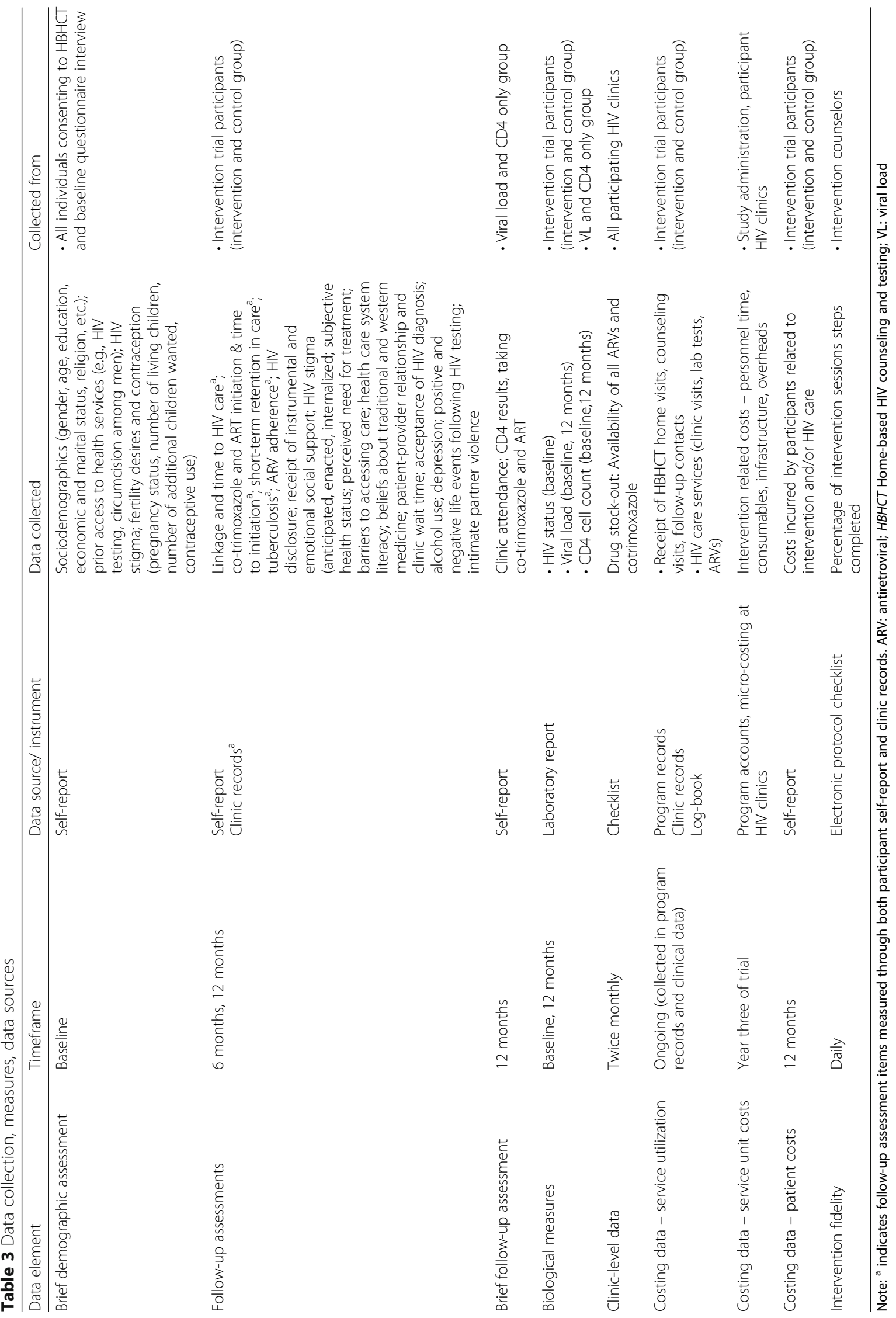


assays, the rapid HIV testing algorithm we will be using in the study is as follows: Determine HIV-1/2 Assay (Alere/ Abbott Laboratories, Chiba, Japan) as the first test in the algorithm. If Determine is non-reactive, an HIV-negative result will be reported. For those found reactive with Determine, a second test, HIV 1/2 STAT-PAK (Chembio Diagnostic Systems, Medford, NY, USA) will be performed. If reactive with STAT-PAK, results will be reported as HIV positive. If reactive with Determine and non-reactive with STAT-PAK, both the Determine and STAT-PAK tests will be repeated. If both the repeat Determine and repeat STAT-PAK are reactive, an HIV-positive result will be reported. If both the repeat Determine and repeat STAT-PAK are non-reactive, an HIV-negative result will be reported. If the repeat Determine is reactive and the repeat STAT-PAK result is non-reactive, the sample will be tested using Uni-Gold HIV (Trinity Biotech, Bray, Ireland). If Uni-Gold is reactive, an inconclusive result will be reported and the participant will be re-tested in 14 days. If Uni-Gold is non-reactive, an HIV negative result will be reported. For quality control purposes, a venous blood sample will be taken with every 10th participant who tests HIV negative. Deoxyribonucleic acid polymerase chain reaction tests will be performed on these specimens for quality control.

\section{Primary outcome measures}

- Individual and population HIV VL suppression (baseline and 12 month). We will examine HIV VL suppression at two levels; VL $<200$ and as undetectable VL — defined as HIV RNA <20 copies/ $\mathrm{mL}$. We will also examine changes in VL between baseline and follow-up to determine reductions considered statistically significant-a threefold, or a $0.5 \log _{10}$ copies/mL reduction [44]. Specimens for VL testing will be collected via venous blood draw at baseline and during the 12-month in-home follow-up interview. HIV VL testing will be performed on plasma processed at the Gombe Hospital Laboratory and subsequently sent to Uganda Virus Research Institute (UVRI). HIV VL testing at UVRI will be done using the Roche COBAS AmpliPrep/TaqMan assay (Roche Molecular Systems, Pleasanton, CA) which has a lower limit of detection of 20 copies/mL.

\section{Secondary outcome measures}

Our main secondary outcome measures include HIV care linkage, ART initiation, and short-term retention in care (detailed below) collected through participant self-report at six and 12-month follow-up interviews, supplemented by clinic records and direct observation of participants' HIV clinic cards and ART pill bottles where possible.

- Linkage and time to care (six and 12 months, clinic records). We will assess linkage to care with data on: enrollment in an HIV clinic, having a second HIV clinic visit, and time from HIV testing to enrollment in care at an HIV clinic.

- ART initiation E time to initiation (six and 12 months, clinic records). To assess receipt of HIV treatment we will determine if participants have been prescribed and have taken ART and the time from HIV testing to ART initiation. We will determine the percentage of participants eligible for ART (currently CD4 < 500 until the guidelines [8] to expand eligibility are implemented nationwide), who received antiretrovirals (ARVs) based on their CD4 count taken at the time of HBHCT, as well as the time from diagnosis to ART initiation. If possible, interviewers will directly observe the participant having ARVs at their home and review the patient's HIV clinic record card to confirm self-reported data.

- Short-term retention in care (six and 12 months, clinic records). We will assess the following retention measures that have demonstrated the best discriminatory capacities for predicting viral suppression [45, 46]: a) missed visit count: number of missed visits accrued (count measure) based on scheduled visits determined by $\mathrm{MOH}$ clinical guidelines, b) visit adherence: proportion of kept visits/scheduled visits (kept + missed visits) (continuous measure, range $=0.0-1.0$ ), and c) four-month visit constancy: number of four-month intervals with at least one kept visit (categorical measure, range $=0-3$ ).

\section{Independent variables}

- Sociodemographics (baseline). We will assess participants' gender, age, tribe, educational level, socioeconomic status, employment status, income, religion, gender, marital/partnership status, distance to clinic, and travel time to the clinic.

- Prior use of health services (baseline). We will measure prior use of HIV services, including history of HIV testing, frequency, location, and reasons for health service use in the prior 6 months, male circumcision for men, and location of births for women.

- CD4 cell count (baseline, 12 months). CD4 cell count will be assessed via venous blood draws along with VL at baseline and 12-month follow-up. 
Baseline CD4 values may affect viral suppression success [47]. We did not include CD4 as an outcome measure because according to recommendations by WHO and the United States Department of Health and Human Services, when VL is available, CD4 has little added value for monitoring treatment adherence and efficacy of ART [44, 48].

- Tuberculosis (TB) (six and 12 months, clinic records). We will assess co-infection with TB using questions adapted from the MOH's HIV clinic card. Interviewers will use the participant's clinic card and self-report to record if the participant is TB infected, date of diagnosis, and treatment initiation and regimen.

- ART adherence (six and 12 months). We will assess self-reported adherence to ART using the Adult AIDS Clinical Trials Group scale [49], which includes recall questions about ARVs missed for the previous 4 days prior to the interview, as well as 17 items assessing participants' main barriers to adherence. It has demonstrated construct validity in Uganda and similar settings [50].

- Disclosure of HIV-test results (six and 12 months). We will measure HIV status disclosure with an instrument used in prior research in Uganda and other African countries $[39,51]$. It asks about the disclosure of serostatus to spouses, sexual partners, family members, community members/leaders, physicians, etc. The proportion of participants reporting each disclosure is calculated after eliminating those who say the disclosure was not applicable to them.

- Receipt of instrumental and emotional social support (six and 12 months). We will measure receipt of instrumental and emotional social support using the Social Support Scale [51], which contains six items on emotional support and four items on instrumental social support. This scale was adapted from the Duke-University of North Carolina Functional Social Support Questionnaire [52] and has been validated among PLHIV in rural Rwanda $(\alpha=0.91)$ [53]. In addition, we will determine if participants have a treatment supporter, have attended HIV support groups, or been counseled by an HIV-positive peer educator, and how helpful each of these forms of counseling have been on a four point scale ranging from "not helpful" to "very helpful."

- HIV stigma (baseline, six, and 12 months). At baseline, for those individuals reporting they are HIV negative or do not know their HIV status, we will measure enacted stigma, including nine items on discrimination, stereotypes, and prejudice using Kalichman et al.s [54] AIDS-related stigma scale, which has been validated in South Africa [54]. We will also measure anticipated stigma, asking participants how they would feel if they tested positive, using six items from Earnshaw's [42] anticipated stigma scale and six items from Berger et al's [55] HIV stigma scale. We will calculate the mean of all participants from each village to get a village (cluster)-level HIV stigma score at baseline. At six and 12-month follow up, and at baseline for those who report already knowing they are HIV positive, we will assess the following dimensions of HIV stigma among PLHIV [56]: (a) anticipated stigma using six items from Earnshaw's [42] anticipated stigma scale and two subscales from Berger et al's [55] HIV stigma scale: eight items assessing anticipated stigma related to disclosure and eight items assessing anticipated stigma related to concern with public attitudes about PLHIV; (b) enacted stigma using six items from Earnshaw's scale [42], 16 items from the personalized HIV stigma subscale of Berger et al's [55] scale, as well as items from Sayles et al.'s [57] multidimensional HIV stigma scale: 12 items from the stereotypes subscale and seven items from the social relationships subscale; and (c) internalized stigma using Kalichman et al.s [58] internalized AIDS-related stigma scale. All of these measures have shown good reliability among sub-Saharan African samples $(\alpha=0.73-0.78)$ [59-61].

- Subjective health status (six and 12 months). We will use the 36-item Medical Outcomes Study-HIV short form [62] to assess subjective health status adapted for the Ugandan context [63] and shown to be reliable among PLHIV in rural populations in Uganda $(\alpha>0.70)[63,64]$.

- Perceived need for HIV care/treatment (six and 12 months). We will adapt five items used in a study in Zambia [65] to assess participants' perceived need for HIV treatment. The items assess participants' agreement with statements such as: "You do not need to go to the HIV clinic because you feel healthy" and "You do not want to take any medicine."

- Barriers to care (six and 12 months). We will measure barriers to attending HIV care, including distance/time to the clinic, financial barriers, work/ child care responsibilities, wait times, and concerns with privacy among health workers. We will include 18 items adapted from the LifeWindows Information Motivation Behavioral Skills ART Adherence Questionnaire [66] and Smith et al's [67] scale on engagement and retention in pre-ART HIV care. We will also determine the actual distance and travel time from the participants' home to the HIV clinic 
and for the cost of transportation to the clinic, for those who are able to pay.

- HIV care system literacy. We will use nine items from the Brief Estimate of Health Knowledge and Action HIV Version scale [68] to assess participants' knowledge of the HIV care system, including questions on where they can get treatment, the cost of treatment, treatment eligibility, etc.

- Beliefs about traditional and western medicine (six and 12 months). We will assess beliefs towards western and traditional medicine and use of traditional medicine to treat HIV using items adapted from the treatment denialism subscale of Kalichman's AIDS denialism scale [69].

- Relationship with the clinic provider. We will measure participants' satisfaction with their healthcare provider using 10 items from a scale used in South Africa by Westaway and colleagues [70].

- Acceptance of HIV diagnosis. Three items modified from the Brief COPE Scale [71] will be used to assess participant's acceptance of their HIV diagnosis by asking participants if they have refused to believe their test results since they tested positive.

- Alcohol use. We will assess alcohol use using the Alcohol Use Disorders Identification Test [72], shown reliable among PLHIV in rural Uganda $(\alpha=0.71)$ [73].

- Depression (six and 12 months). We will measure depression symptoms using a modified 20-item version of the Center for Epidemiological Studies scale [74], which has been shown to be reliable in a rural Ugandan sample $(\alpha=0.90)$ [75].

- Positive and negative life events following HBHCT (six and 12 months). We will include items to assess life events following HIV testing using items by Grinstead et al. [76] and used in our prior work in Uganda [39]. The scale assesses the occurrence of life events since diagnosis, such as the strengthening of a relationship, breakup of a relationship, physical abuse by a sexual partner, and neglect by family.

- History of intimate partner violence (six and 12 months). Items assessing experience of intimate partner violence originating from the Conflict Tactics Scale [77] and included in the WHO multicountry study [78] will be included. The scale includes 10 questions asking participants if they experienced any emotional (e.g., has he/she ever insulted you or made you feel bad about yourself?), physical (e.g., has he/she ever kicked you, dragged you, or beaten you up), and sexual violence (e.g., has he/she physically forced you to have sexual intercourse when you did not want to?) from a partner ever, and in the prior 12 months.
- Intervention contamination (six and 12 months). We will ask participants what type of counseling they received and from whom (to assess potential contamination) and the number of HIV-positive household members and if they are in care and on treatment. We will keep records on the number of and duration of intervention sessions each intervention arm participant actually received.

- Clinic level factors (twice monthly). We will monitor ART and cotrimoxazole stock outs at the participating clinics and hospital, where participants will most frequently visit to pick up their medications, on a twice-monthly basis through use of a checklist that includes of all drugs routinely prescribed for HIV-positive individuals.

\section{Cost measures}

We will collect cost data to determine total intervention cost under enhanced linkage and standard-of-care arms, as well as the costs of HIV-related health services or other costs incurred up to 12 months following the receipt of $\mathrm{HBHCT}$, using methods previously developed by the research team $[9,79]$. Costs will be assessed in three categories: (i) direct costs of HBHCT \& linkage activities, (ii) HIV care costs incurred as a consequence of HBHCT \& linkage activities, and (iii) time-costs and out-of-pocket expenses incurred by individuals receiving the intervention. For the direct costs of HBHCT and linkage activities, data on personnel time and consumables will be extracted from staff activity logs, and data on training costs, transportation, and management/administrative overheads will be collected from program records. For the costs of additional HIV care and laboratory services incurred as a consequence of the intervention, costing will be undertaken at HIV clinics to establish unit costs for clinic visits, ART and other medicines, and routine laboratory tests. The number and timing of HIV-related services received by study participants will be collected from clinic records. Wages and other input prices will be based on public sector values to ensure that results are applicable to routine program settings. During the 12-month follow-up interviews we will include questions to determine costs incurred by study participants in relation to receipt of the intervention and HIV care (including time costs, travel costs, and any out-of-pocket payments related to HIV care).

\section{Monitoring intervention fidelity}

Counselors will use electronic intervention protocols programmed on tablets to guide them in completing the components of each intervention session. For each step they will document its completion by selecting potential outcomes or typing in a description, e.g., "primary barrier is fear of being seen at clinic;" "no noted potential 
adverse events." This will allow us to capture the percentage of intervention protocol steps that the counselor delivered. We have used this method to monitor intervention fidelity in previous intervention research in Uganda [80].

\section{Data analysis approach}

Univariate statistics will be conducted on primary and secondary endpoints as well as covariates to describe the sample characteristics for the two study arms. Attrition in the two arms will be compared. Multiple imputation technique will be implemented and sensitivity analysis will be conducted.

To assess the primary outcome (the proportion of participants who are virally suppressed at 12-months) and secondary outcomes (linkage to care, time to care, receipt of opportunistic infection prophylaxis, and initiation of ART among those eligible for ART at baseline), linear mixed models and nonlinear mixed models will be applied. Information criterion such as Akaike information criterion or Bayesian information criterion will be used for model selection.

In evaluating the intervention effectiveness mechanisms, and mediators and moderators, we will use structural equation modeling to estimate the causal effects using growth mixture modeling to account for the longitudinal and clustered data. To assess an existence of moderators of an intervention effect, covariates such as disclosure, social support, HIV stigma, adherence, distance from the clinic, CD4 count, having other HIVpositive household members, number and duration of intervention sessions received, village-level stigma towards PLHIV, etc. will be included. A nonlinear mixed model will be fitted independently for each covariate plus the treatment variable and an interaction term between the covariate and the treatment.

To examine the natural history of stages in the HIV care continuum, we will use the control group to identify barriers to and facilitators of linkage to care and treatment, retention, and viral suppression, potential predictors include: sociodemographics, HIV stigma, disclosure, etc. Stepwise forward selection method from both logistic regression model and general linear model will be used to identify potential significant predictors for our analyses. Predictors that are selected from the stepwise method will be included in our mixed models.

\section{Sample size justification and power analysis}

The sampling scheme is stratified clustering with matched-pair sampling. Within each area, we pair matched clusters (villages) by distance to the HIV clinic and randomly assigned one village to be in the intervention arm. We have ensured that villages assigned to different study conditions are not within $4 \mathrm{~km}$ ( $1 \mathrm{~h}$ by foot) of each other to avoid possible discussions between different arms of participants.

We have powered the study to detect differences between the intervention and the standard-of-care for the primary outcome of HIV viral suppression $(<20$ copies $/ \mathrm{ml}$ ) as well as intermediate outcomes: linkage to care and time to care, and, among those eligible for ART: time to and receipt of ART. Using census data, HBHCT acceptance rates from our own and other prior studies [22], HIV prevalence and prior knowledge of HIV status in this area from the recent prevalence survey [17], and estimating a $92 \%$ acceptance rate for enrollment of eligible participants into the study we calculated that the average cluster size (number of newly-diagnosed PLHIV per village who enroll in the study) will be 14 (range 8 to 37). In calculating the necessary clusters, type I error rate, desired power of tests, measure of effectiveness between the intervention and control group, cluster size, and coefficient of variation $(\mathrm{k})$ or intraclass correlation coefficient are needed. We estimated viral suppression at 12-month follow-up among those eligible for ART at baseline to be $55 \%$ in the standard-of-care arm and $75 \%$ in the intervention arm. This estimate takes into account 12-month viral suppression success rates from SSA public sector ART programs [45, 81-84] and our own [23,39] and others' data on intermediate outcomes of linkage to care and ART initiation [22, 28]. With a type I error rate of 0.05 (two sided), average cluster size of 14 , power of $80 \%, 12 \%$ difference in outcomes, and coefficient of variation of 0.24 , we need 40 clusters after accounting for a $18 \%$ attrition rate for the follow-up. We expect that the 40 clusters will yield approximately 600 PLHIV participants across the two study arms.

\section{Ethics}

Approval of all study procedures has been obtained from the institutional review boards at San Diego State University and Makerere School of Public Health. Study approval has also been received from the Uganda National Council for Science and Technology. We will obtain written informed consent for participation in HIV testing and the brief baseline questionnaire interview. For individuals who want HIV testing, but do not want to participate in the brief questionnaire interview, verbal informed consent with a waiver for written consent will be obtained. Separate consent forms will be used to obtain written informed consent for all participants enrolled in the intervention trial or CD4 and VL Only arm.

We expect that the overall risk:benefit ratio will be favorable to patients participating in the study, and the risks related to HBHCT are not greater than the risks associated with the standard-of-care health system procedures related to home-based health services, which is commonplace in this rural Ugandan setting. Among the 
main risks of the study, are minimal physical risks associated with fingerstick and venous blood draws (bruising, bleeding, discomfort, lightheadedness, and potential infection), which are not greater than the risks incurred from normal medical blood testing procedures. We will minimize these risks by hiring qualified staff who also have experience in phlebotomy and finger stick blood collection to perform these procedures.

There is also the potential for breaches in confidentiality related to collected data (detailed contact information of patients, interviewer-administered computerized questionnaires, data extracted from medical records, HIV results, CD4 and VL test results). To reduce the potential for recorded data to identify the participant, we will (a) use unique identifiers (random 6 digit study identification (ID) numbers) instead of medical ID/record numbers or participant names to identify all data; (b) store of the list that links the participants' names to their unique identifiers in a locked, secure location; (c) store participant contact details in a locked cabinet separate from the list that links participant names to study ID numbers and it will not contain the unique study ID number, (d) use encryption protocols and password protection for all data collected and/or stored electronically and encryption procedures to securely transfer data, and (e) train all study staff in the importance of and procedures for protecting participants' confidentiality.

Participants in our study may experience discomfort while discussing sensitive information during intervieweradministered computerized questionnaires (e.g., domestic violence) and intervention counseling sessions (e.g., barriers to attending HIV care). These risks will be mitigated through clearly informing patients of their right to refuse to answer any question or engage in any discussion that makes them uncomfortable and providing adequate training to counselors and interviewers in navigating sensitive discussions. For participants receiving the intervention, the possibility of experiencing an unforeseen unintended negative consequence from participating in the intervention (e.g., negative reactions to their disclosure of HIV status). This risk will be mitigated by training counselors delivering the intervention to conduct counseling sessions only in private settings and to use judgement in which strategies are appropriate for participants based on their personal circumstances. Finally, there is potential for public disclosure of the HIV test result. However, HIV test results, like all other data, will only be identified by a randomly generated study ID number. We discuss ethical concerns and protections for participant confidentiality further in the discussion section.

\section{Discussion}

We hypothesize that compared to individuals receiving standard-of-care, or paper-based referrals, individuals receiving the PATH/Ekkubo intervention will be more likely to be virally suppressed 12 months after HBHCT, and demonstrate shorter time to HIV care and ART initiation among those eligible. Individuals receiving the PATH intervention will receive multiple follow-up counseling sessions aimed to address barriers to HIV care. This study will indicate whether enhanced linkage to care counseling found effective in improving linkage to HIV care during facility-based HIV testing in an urban population [39] can similarly improve linkage to HIV care, and subsequently reduce $\mathrm{VL}$, when integrated into HBHCT in a rural setting. This study will also provide further information on the cost-effectiveness of this approach compared to providing standard-of-care paperbased referrals during HBHCT.

The evaluation of this intervention has the ability to advance science and improve public health service delivery in several ways. To date, few randomized controlled trials have assessed interventions to improve linkage to care in the context of HBHCT, leaving a gap in our understanding of the best approaches to promote linkage to HIV care and treatment in HBHCT in SSA countries. For those in the intervention arm, it is anticipated that this study will help patients reduce barriers to accessing HIV care by providing strategies and resources to overcome stigma and other barriers, as well as the requisite knowledge, motivation, and self-efficacy needed to do so. If found effective, our study will provide information on the mechanisms through which our intervention influenced linkage behavior, which will be useful in the development of future interventions. Specifically, we will test the hypothesis based on prior research, that social support will have a protective effect against enacted and internalized stigma [32, 33, 38] and additionally test the subsequent effects on linkage to care, receipt of HIV treatment, and viral suppression. In addition, by treating the control-arm as a natural history control, our study will further contribute to the scientific literature on barriers to and facilitators of linkage to care and treatment and HIV viral suppression. This data, together with our findings on costing, have the potential to inform the integration of cost-effective avenues for follow-up and the delivery of support for HBHCT in Uganda and similar settings.

\section{Confidentiality and ethical considerations}

Conducting a CRCT in the setting of HBHCT has unique ethical challenges surrounding confidentiality of participants' HIV status. Given the nature of our intervention, which includes multiple home visits for PLHIV, efforts to minimize the risk of participants being identified as HIV positive in their community or families through participation in our study are paramount. In addition to commonly employed safeguards to protect 
participant confidentiality, such as the use of unique identifiers linked to personally identifying information and the careful storage and transfer of sensitive data (as described previously), we describe here issues of confidentiality and ethics specific to conducting research with PLHIV during HBHCT, which may also be relevant to other community-based trials that involve PLHIV, and how we've aimed to address them.

With research involving PLHIV, there is always small risk of public disclosure. In our study, home visits from counselors could increase risk of public disclosure. While we think it is unlikely that visits from counselors in it of itself would signal one's HIV-positive status to others in the community, as visits from counselors and various health workers are common in Uganda for a variety of healthcare reasons (e.g., family planning, postnatal care, malaria care, immunizations), we will minimize this risk in several ways. Firstly, when counselors do home visits to HIV-positive participants for the intervention trial, they will also visit other homes nearby.

Secondly, only those who have tested HIV positive and are enrolled in the intervention trial will know that the intervention trial is about linkage to HIV care. Participants in the baseline interview and HIV testing and those who only want HIV testing will not be informed about the intervention trial and thus will not know that those who are positive and in the intervention trial will be getting home visits. The baseline assessment itself will be framed more broadly as a survey on health care access and will include questions on other health service uptake beyond HIV (e.g., family planning) to mask the study's purpose. Masking the purpose of the study requires that we consent participants for the intervention trial after they have learned they are HIV positive.

In addition to potential breaches in confidentiality among community members, counselor visits also have the risk of family members or others overhearing discussions in which sensitive information is being addressed. Training counselors to ensure privacy during home visits, as well as training counselors to use their judgment in rescheduling a home visit if privacy is compromised will minimize this risk.

Although this risk is considered to be low, some strategies that patients elect to implement in order to engage in HIV care may potentially have unintended negative consequences on the study participant or others. To minimize this risk, counselors will be trained to respect patients' decisions and their comfort levels with HIV care linkage strategies and goals, emphasizing the decision to engage in HIV care, disclose one's HIV status to others, and seek social support will be a personal one of the patient. When assessing linkage behavior, all components of a patient's physical health, mental health, and social situation will be considered when discussing linkage strategies, including the threat of domestic violence. A variety HIV care linkage strategies will be discussed with each participant to minimize the possibility that a particular plan to access care may place a patient in any danger. Participants will choose the one least likely to cause negative consequences. Counselors will screen for the threat of domestic violence when deciding on a disclosure plan and linkage strategies during the first counseling session, and will conduct a brief risk assessment for domestic violence at every subsequent meeting with participants. Should participants require additional support, counselors will refer participants to relevant staff at the clinic or hospital, who will be equipped to provide appropriate referrals as necessary to study participants for additional counseling, substance abuse issues, domestic abuse, and the threat of violence when these issues arise. Participants who disclose potential abuse (both men and women) will be immediately referred to community and/or onsite services equipped to manage situations of domestic abuse when available or to higher levels when unavailable. Immediate access to crisis intervention and emergency health services, including referrals for safe shelter, are available through the hospital as needed.

\section{Limitations}

Among the study's limitations is the threat for contamination between intervention and control villages. However, randomizing at the level of the village and having villages assigned to different study arms not geographically close to one another should minimize this threat. We will monitor potential contamination by asking all study participants what kind of linkage counseling they received and from whom. Interviewers conducting the follow-up assessments will be blinded to study condition, however, un-blinding is possible if participants discuss the intervention with the interviewer. We will monitor this by debriefing interviewers. In addition to individual-level data, we will collect clinic-level data; cross-classification may occur if participants seek HIV care at a clinic other than the one nearest to them. However, our data analysis approach can accommodate cross-classification. Finally, in order for participants to be eligible for participation, they must be willing to accept home visits, which may limit the generalizability of the findings. However, we judge this to be only slightly more than in any study which tracks participants via home visits, and from prior studies, we have found that very few patients decline to participate due to the possibility of being visited at home.

\section{Conclusion}

If the PATH/Ekkubo intervention is found effective, the anticipated public health benefits include improving 
individual health by reducing the time in which participants are linked to care. Timely linkage to care improves treatment outcomes and reduces mortality rates [85-87]. Participation in our study in itself regardless of study condition provides individuals the opportunity to learn their HIV status, and receive diagnosis and treatment for HIV that would have otherwise gone unnoticed and untreated. Moreover, through ART's effect on VL, increasing the number of PLHIV who are in HIV care and receiving ART will have a secondary outcome of the prevention of ongoing HIV transmission to others [48]. Our study will further add to the scientific literature on the potential population-level health benefits of behavioral interventions, by assessing population-level VL as a study outcome.

\begin{abstract}
Abbreviations
AACTG: Adult AIDS Clinical Trials Group; AIDS: Acquired Immunodeficiency Syndrome; ART: Antiretroviral therapy; ARVs: Antiretroviral medications; AUDIT: Alcohol use disorders identification test; CRCT: Cluster randomized controlled trial; HBHCT: Home-based HIV counseling and testing; HIV: Human immunodeficiency virus; ID: Identification; $\mathrm{MOH}$ : Ministry of health; PATH Study: Providing access to HIV care study; PLHIV: People living with HIV; SSA: Sub-Saharan Africa; TB: Tuberculosis; UVRI: Uganda Virus Research Institute; VHT: Village health team; VL: Viral load; WHO: World Health Organization
\end{abstract}

\section{Acknowledgements}

We acknowledge the contributions of Butambala, Mpigi, Mityana, and Gomba Health Districts and the participating health clinics in the implementation of this study and thank them for their support. We owe particular gratitude to Gombe Hospital for being the field-base for our study and to the hospital management for assisting with study logistics. We also thank the hospital lab for receiving, processing, and storing our samples. This study would not be possible without the support and assistance with community mobilization from the VHTs and local community stakeholders. We would also like to acknowledge the UVRI for their role in performing the HIV VL testing for the study.

\section{Funding}

This study is supported by the National Institute of Mental Health (NIMH) grant number R01MH106391, awarded to Susan M. Kiene and Rhoda K. Wanyenze.

\section{Availability of data and materials}

The datasets generated from the current study will not be made publicly available. However, 3 years after the completion of the study, the fully de-identified data will be made available to researchers and their students at the Principal Investigators' institutions: San Diego State University and the Makerere University School of Public Health. On a case-by-case basis, data may be made available to researchers at other institutions.

\section{Authors' contributions}

SMK and RKW conceived and designed the design and are overseeing project implementation. SCK provided input on the study design and intervention protocol. SMK, RKW, and KMS led the drafting of the manuscript. NAM provided input on the overall study design, and developed the cost-effectiveness analysis section. KMS and RN contributed to project implementation. CDL developed the sample size justification and statistical methods. $\mathrm{HL}$ and MHB provided input on overall study design, and provided input on community mobilization methods and the integration of the study into the context of home-based HIV counseling and testing. MHB trained the intervention counselors. All authors contributed to the review and revision of the article, and approved the final manuscript.

\section{Competing interests}

The authors declare that they have no competing interests.

\section{Consent for publication}

Not applicable.

\section{Ethics approval and consent to participate}

The study was approved by San Diego State University and Makerere University School of Public Health. Permission for the study has also been received from the Uganda National Council for Science and Technology. Informed consent will be obtained from all participants receiving HBHCT, and additional consent will be obtained before any additional data collection and intervention participation.

\section{Disclamer}

The opinions in this article are those of the authors and should not be construed to represent the positions of the the National Institutes of Health, the United States, or the U.S. Federal Government.

\section{Publisher's Note}

Springer Nature remains neutral with regard to jurisdictional claims in published maps and institutional affiliations.

\section{Author details}

'Division of Epidemiology and Biostatistics, Graduate School of Public Health, San Diego State University, 5500 Campanile Drive (MC-4162), San Diego, CA 92182, USA. ${ }^{2}$ Department of Psychology, University of Connecticut, Storrs, CT, USA. ${ }^{3}$ Division of Epidemiology and Biostatistics, Graduate School of Public Health, San Diego State University, San Diego, CA, USA. ${ }^{4}$ Department of Global Health and Population, Harvard T. H. Chan School of Public Health, Boston, MA, USA. ${ }^{5}$ Department of Disease Control and Environmental Health, Makerere University School of Public Health, Kampala, Uganda. ${ }^{6}$ Department of Mathematics and Statistics, San Diego State University, San Diego, CA, USA. 'Formerly: Department of Global Health, University of Washington, Seattle, WA, USA. ${ }^{8}$ Gombe Hospital, Gombe, Uganda.

Received: 2 June 2017 Accepted: 9 June 2017

Published online: 03 July 2017

\section{References}

1. UNAIDS. 90-90-90: An ambitious treatment target to help end the AIDS epidemic. Geneva: UNAIDS; 2014.

2. World Health Organization. Consolidated Guideliens on the Use of Antiretroviral Drugs for Treating and Preventing HIV Infection: Recommendation for a Public Health Approach. 2nd ed. Geneva: WHO; 2016.

3. UNAIDS. AIDS Data: UNAIDS 2016 Reference. Geneva: UNAIDS. 2016. Retrieved from: http://www.unaids.org/sites/default/files/media_asset/2016AIDS-data_en.pdf.

4. Kranzer K, Govindasamy D, Ford N, Johnston V, Lawn SD. Quantifying and addressing losses along the continuum of care for people living with HIV infection in sub-Saharan Africa: a systematic review. J Int AIDS Soc. 2012; 15(2):17383.

5. Govindasamy D, Ford N, Kranzer K. Risk factors, barriers and facilitators for linkage to antiretroviral therapy care: a systematic review. AIDS. 2012;26(16): 2059-67.

6. Mugglin C, Estill J, Wandeler G, Bender N, Egger M, Gsponer T, et al. Loss to programme between HIV diagnosis and initiation of antiretroviral therapy in sub-Saharan Africa: Systematic review and meta-analysis. Tropical Med Int Health. 2012;17(12):1509-20.

7. Rosen S, Fox MP. Retention in HIV care between testing and treatment in sub-Saharan Africa: a systematic review. PLoS Med. 2011;8(7):e1001056.

8. Uganda Ministry of Health. Consolidated Guidelines for Prevention and Treatment of HIV in Uganda. Kampala: Uganda Ministry of Health; 2016.

9. Menzies N, Abang B, Wanyenze R, Nuwaha F, Mugisha B, Coutinho A, et al. The costs and effectiveness of four HIV counseling and testing strategies in Uganda. AIDS. 2009;23(3):395-401.

10. Mulogo EM, Batwala V, Nuwaha F, Aden AS, Baine OS. Cost effectiveness of facilitiy and home based HIV voluntary counseling and testing strategies in rural Uganda. Afr Health Sci. 2013;13(2):423-9.

11. Leach-Lemens C, Owuor J. Scaling up HIV testing and counselling towards universal access: what works in resource-limited settings. HIV/AIDS Treatment in Practice; 2009. p. 2-10. Retrieved from: http://www.aidsmap.com/ cms1330557.aspx.

12. MacPherson P, Lalloo DG, Webb EL, Maheswaran H, Choko AT, Makombe SD, et al. Effect of optional home initiation of HIV care following HIV self-testing on antiretroviral therapy initiation among adults in Malawi: a randomized clinical trial. J Am Med Assoc. 2014;312(4):372-9. 
13. Desai MA, Okal D, Rose C, Ndivo R, Williams T, Otieno F. Point-of-Care CD4 (Pima) impact on linkage to care with home-based HIV testing, Kenya. In: Conference on retroviruses and opportunistic infections: Feb 23-26 2015; Seattle, WA; 2015.

14. Barnabas RV, van Rooyen $H$, Tumwesigye E, Brantley J, Baeten JM, van Heerden A, et al. Uptake of antiretroviral therapy and male circumcision after community-based HIV testing and strategies for linkage to care versus standard clinic referral: a multisite, open-label, randomised controlled trial in South Africa and Uganda. Lancet HIV. 2016;3(5):e212-20.

15. Geng EH, Bangsberg DR, Musinguzi N, Emenyonu N, Bwana MB, Yiannoutsos $C T$, et al. Understanding reasons for and outcomes of patients lost to followup in antiretroviral therapy programs in Africa through a sampling-based approach. J. Acquir. Immune Defic. Syndr. 2010;53(3):405-11.

16. Bwambale FM, Ssali SN, Byaruhanga S, Kalyango JN, Karamagi CA. Voluntary HIV counselling and testing among men in rural western Uganda: implications for HIV prevention. BMC Public Health. 2008;8:263.

17. Uganda Ministry of Health, ICF International. Uganda AIDS Indicator Survey (AIS) 2011. Kampala, Calverton, MD: Uganda Ministry of Health and ICF International; 2012.

18. Kiene SM, Sileo K, Wanyenze RK, Lule H, Bateganya MH, Jasperse J, et al. Barriers to and acceptability of provider-initiated HIV testing and counselling and adopting HIV-prevention behaviours in rural Uganda: a qualitative study. J Health Psychol. 2015;20(2):173-87.

19. Saleem H, Kyeyagalire R, Lunsford SS. Patient and provider perspectives on improving the linkage of HIV-positive pregnant women to long-term HIV care and treatment in eastern Uganda. African J AIDS Res. 2014;13(1):45-51.

20. Marum E. Innovations, issues and debates in HIV testing and counselling. In: HIV/AIDS Implementers Conference: June 13, 2009; Windhoek, Namibia; 2009.

21. Shumba CS, Atuhaire L, Memiah P, Atukunda R. Assessment of community mobilization and home-based HIV counselling and testing offered by health facilities in rural Uganda. Afr J Reprod Health. 2013;17(4 Spec):171-6.

22. Tumwesigye E, Wana G, Kasasa S, Muganzi E, Nuwaha F. High uptake of home-based, district-wide, HIV counseling and testing in Uganda. AIDS Patient Care STDs. 2010;24(11):735-41.

23. Kyaddondo D, Wanyenze R, Kinsman J, Hardon A. Home-based HIV counseling and testing: Client experiences and perceptions in Eastern Uganda. BMC Public Health. 2012;12(1):966.

24. Naik R, Doherty $T$, Jackson D, Tabana H, Swanevelder S, Thea DM, et al. Linkage to care following a home-based HIV counselling and testing intervention in rural South Africa. J Int AIDS Soc. 2015;18:19843.

25. Iwuji CC, Orne-Gliemann J, Larmarange J, Okesola N, Tanser F, Thiebaut R, et al., group ATt. Uptake of Home-Based HIV Testing, Linkage to Care, and Community Attitudes about ART in Rural KwaZulu-Natal, South Africa: Descriptive Results from the First Phase of the ANRS 12249 TasP ClusterRandomised Trial. PLoS Med. 2016;13(8):e1002107.

26. Barnabas RV, van Rooyen H, Tumwesigye E, Murnane PM, Baeten JM, Humphries $\mathrm{H}$, et al. Initiation of antiretroviral therapy and viral suppression after home HIV testing and counselling in KwaZulu-Natal, South Africa, and Mbarara district, Uganda: a prospective, observational intervention study. Lancet HIV. 2014;1(2):e68-76.

27. Medley A, Ackers M, Amolloh M, Owuor P, Muttai H, Audi B, et al. Early uptake of HIV clinical care after testing HIV-positive during home-based testing and counseling in Western Kenya. AIDS Behav. 2013;17(1):224-34.

28. Bigogo G, Amolloh M, Laserson KF, Audi A, Aura B, Dalal W, et al. The impact of home-based HIV counseling and testing on care-seeking and incidence of common infectious disease syndromes in rural western Kenya. BMC Infect Dis. 2014;14(1):376.

29. Tumwebaze H, Tumwesigye E, Baeten JM, Kurth AE, Revall J, Murnane PM, et al. Household-based HIV counseling and testing as a platform for referral to HIV care and medical male circumcision in Uganda: a pilot evaluation. PLoS One. 2012;7(12):e51620.

30. van Rooyen H, Barnabas RV, Baeten JM, Phakathi Z, Joseph P, Krows M, et al. High HIV testing uptake and linkage to care in a novel program of homebased HIV counseling and testing with facilitated referral in KwaZulu-Natal, South Africa. J Acquir Immune Defic Syndr. 2013;64(1):e1-8.

31. Ware NC, Wyatt MA, Asiimwe S, Turyamureeba B, Tumwesigye E, van Rooyen $\mathrm{H}$, et al. How home HIV testing and counselling with follow-up support achieves high testing coverage and linkage to treatment and prevention: a qualitative analysis from Uganda. J Int AIDS Soc. 2016;19(1):20929.

32. Stangl AL, Lloyd JK, Brady LA, Holland CE, Baral S. A systematic review of interventions to reduce HIV-related stigma and discrimination from 2002 to 2013: how far have we come? J Int AIDS Soc. 2013;16(2):18734.
33. Tshabalala J, Visser M. Developing a cognitive behavioural therapy model to assist women to deal with HIV and stigma. South African J Psychol. 2011; 41(1):17-28.

34. Muhamadi L, Tumwesigye NM, Kadobera D, Marrone G, Wabwire-Mangen F, Pariyo $\mathrm{G}$, et al. A single-blind randomized controlled trial to evaluate the effect of extended counseling on uptake of pre-antiretroviral care in Eastern Uganda. Trials. 2011;12:184.

35. Otieno V, Marima R, Odhiambo J, Penner J, Ooko H, Agot K, et al. Improving enrollment and retention of youth into HIV services: lessons learned from Kisumu, Kenya. In: XVII International AIDS Conference: August 3-8, 2008 2008; Mexico City, Mexico; 2008.

36. Duff P, Kipp W, Wild TC, Rubaale T, Okech-Ojony J. Barriers to accessing highly active antiretroviral therapy by HIV-positive women attending an antenatal clinic in a regional hospital in western Uganda. J Int AIDS Soc. 2010;13(1):37.

37. Hatcher A, Turan J, Leslie H, Kanya L, Kwena Z, Johnson M, et al. Predictors of linkage to care following community-based HIV counseling and testing in rural Kenya. AIDS Behav. 2012;16(5):1295-307.

38. Takada S, Weiser SD, Kumbakumba E, Muzoora C, Martin JN, Hunt PW, et al. The dynamic relationship between social support and HIV-related stigma in rural Uganda. Ann Behav Med. 2014;48(1):26-37.

39. Wanyenze RK, Kamya MR, Fatch R, Mayanja-Kizza H, Baveemo S, Szekeres G, Bangsberg DR, Coates T, JA H: Abbreviated HIV counselling and testing and enhanced referral to care in Uganda: a factorial randomised controlled trial. Lancet Glob Health 2013, 1(3):e137-e145.

40. Doherty M. New Directions in the 2015 Consolidated ARV Guidelines Update. In: Eighth International AIDS Society Conference on HIV Pathogenesis, Treatment and Prevention, July 19, 2015, Vancouver, Canada. 2015.

41. Uganda Ministry of Health. National Implementation guidelines for HIV Counselling and Testing in Uganda. Kampala: Ministry of Health. 2010. Retrieved from: http://sustainuganda.org/sites/sustainuganda.org/files/GL_ HTC_National_Implementation_Guidelines_Uganda\%28Dec2010\%29_0.pdf.

42. Earnshaw VA, Smith LR, Chaudoir SR, Amico KR, Copenhaver MM. HIV Stigma Mechanisms and Well-Being among PLWH: A Test of the HIV Stigma Framework. AIDS Behav. 2013;17(5):1785-95.

43. World Health Organization. WHO Recommendations to Assure HIV Testing Quality. Geneva: World Health Organization; 2015. Retrieved from: http:// www.who.int/hiv/pub/toolkits/policy-hiv-testing-quality-assurance/en/.

44. Panel on Antiretroviral Guidelines for Adults and Adolescents. Guidelines for the use of antiretroviral agents in HIV-1-infected adults and adolescents. Washington DC: Department of Health and Human Services; 2012. Retrieved from: http://www.aidsinfo.nih.gov/ContentFiles/AdultandAdolescentGL.pdf.

45. Crawford TN, Sanderson WT, Thornton A. A Comparison Study of Methods for Measuring Retention in HIV Medical Care. AIDS Behav. 2013;17(9):3145-51.

46. Mugavero MJ, Davila JA, Nevin CR, Giordano TP. From access to engagement: measuring retention in outpatient HIV clinical care. AIDS Patient Care STDs. 2010;24(10):607-13.

47. Costiniuk CT, Sigal A, Jenabian MA, DW NP. Short communication: lower baseline CD4 count is associated with a greater propensity toward virological failure in a cohort of South African HIV patients. AIDS Res Hum Retrovir. 2014;30(6):531-4.

48. World Health Organization. Consolidated guidelines on the use of antiretroviral drugs for treating and preventing HIV infection. Geneva: WHO; 2013.

49. Chesney MA, Ickovics JR, Chambers DB, Gifford AL, Neidig J, Zwickl B, et al., Adherence Working Group Of The Outcomes Committee Of The Adult Aids Clinical Trials G. Self-reported adherence to antiretroviral medications among participants in HIV clinical trials: The AACTG Adherence Instruments. AIDS Care. 2000;12(3):255-66.

50. Oyugi JH, Byakika-Tusiime J, Charlebois ED, Kityo C, Mugerwa R, Mugyenyi P, et al. Multiple validated measures of adherence indicate high levels of adherence to generic HIV antiretroviral therapy in a resource-limited setting. J Acquir Immune Defic Syndr. 2004;36(5):1100-2.

51. Antelman G, Smith Fawzi MC, Kaaya S, Mbwambo J, Msamanga Gl, Hunter DJ, et al. Predictors of HIV-1 serostatus disclosure: a prospective study among HIV-infected pregnant women in Dar es Salaam, Tanzania. AIDS. 2001;15(14):1865-74.

52. Broadhead WE, Gehlbach SH, Gruy FV, Berton HK. The Duke-UNC functional social support questionnaire: Measurement of social support in family medicine patients. Med Care. 1988;26(7):709-23.

53. Epino HM, Rich ML, Kaigamba F, Hakizamungu M, Socci AR, Bagiruwigize $E$, et al. Reliability and construct validity of three health-related self-report scales in HIV-positive adults in rural Rwanda. AIDS Care. 2012;24(12):1576-83. 
54. Kalichman SC, Simbayi LC, Jooste S, Toefy Y, Cain D, Cherry C, et al. Development of a brief scale to measure AIDS-related stigma in South Africa. AIDS Behav. 2005;9(2):135-43.

55. Berger BE, Ferrans CE, Lashley FR. Measuring stigma in people with HIV: psychometric assessment of the HIV stigma scale. Res Nurs Health. 2001; 24(6):518-29.

56. Earnshaw VA, Chaudoir SR. From Conceptualizing to Measuring HIV Stigma: A Review of HIV Stigma Mechanism Measures. AIDS Behav. 2009;13(6):1160-77.

57. Sayles J, Hays R, Sarkisian C, Mahajan A, Spritzer K, Cunningham W. Development and psychometric assessment of a multidimensional measure of internalized HIV stigma in a sample of HIV-positive adults. AIDS Behav. 2008;12(5):748-58.

58. Kalichman SC, Simbayi LC, Cloete A, Mthembu PP, Mkhonta RN, Ginindza T. Measuring AIDS stigmas in people living with HIV/AIDS: the Internalized AIDS-Related Stigma Scale. AIDS Care. 2008;21(1):87-93.

59. Lyimo RA, Stutterheim SE, Hospers HJ, de Glee T, van der Ven A, de Bruin M. Stigma, disclosure, coping, and medication adherence among people living with HIV/AIDS in Northern Tanzania. AIDS Patient Care STDs. 2014;28(2):98-105.

60. Evangeli M, Newell ML, Richter L, McGrath $\mathrm{N}$. The association between selfreported stigma and loss-to-follow up in treatment eligible HIV positive adults in rural Kwazulu-Natal, South Africa. PLoS One. 2014;9(2):e88235.

61. Tsai AC, Bangsberg DR, Kegeles SM, Katz IT, Haberer JE, Muzoora C, et al. Internalized stigma, social distance, and disclosure of HIV seropositivity in rural Uganda. Ann Behav Med. 2013;46(3):285-94.

62. Sherbourne CD, Stewart AL. The MOS social support survey. Soc Sci Med. 1991;32(6):705-14

63. Mast TC, Kigozi G, Wabwire-mangen F, Black R, Sewankambo N, Serwadda D, et al. Measuring quality of life among HIV-infected women using a culturally adapted questionnaire in Rakai district, Uganda. AIDS Care. 2004;16(1):81-94.

64. Babikako H, Neuhauser D, Katamba A. Feasibility, reliability and validity of health-related quality of life questionnaire among adult pulmonary tuberculosis patients in urban Uganda: cross-sectional study. Health Qual Life Outcomes. 2010;8:93. doi:10.1186/1477-7525-8-93.

65. Fox M, Mazimba A, Seidenberg P, Crooks D, Sikateyo B, Rosen S. Barriers to initiation of antiretroviral treatment in rural and urban areas of Zambia: a cross-sectional study of cost, stigma, and perceptions about ART. J Acquir Immune Defic Syndr. 2010;13(1):8.

66. The LifeWindows Project Team. The LifeWindows Information Motivation Behavioral Skills ART Adherence Questionnaire (LW-IMB-AAQ). Center for Health, Intervention, and Prevention. University of Connecticut; 2006. Retrieved from: http://chipcontent.chip.uconn.edu/chipweb/documents/ Research/F_LWIMBARTQuestionnaire.pdf.

67. Smith L. Engagement and retention in pre-ART HIV care: KwaZulu-Natal South Africa. Center for Health, Intervention, and Prevention. University of Connecticut; 2011. Retrieved from: http://chipcontent.chip.uconn.edu/wpcontent/uploads/2015/11/PreARTRetention.pdf.

68. Osborn C, Davis T, Bailey S, Wolf M. Health Literacy in the Context of HIV Treatment: Introducing the Brief Estimate of Health Knowledge and Action (BEHKA)—HIV Version. AIDS Behav. 2010;14(1):181-8.

69. Kalichman S, Eaton L, Cherry C. "There is no proof that HIV causes AIDS": AIDS denialism beliefs among people living with HIV/AIDS. J Behav Med. 2010;33(6):432-40.

70. Westaway MS, Rheeder P, van Zyl DG. Development and testing of a 25-item patient satisfaction scale for black South African diabetic outpatients. Curationis. 2002;25(3):68-75

71. Carver CS. You want to measure coping but your protocol's too long: Consider the Brief COPE. Int. J. Behav. Med. 1997:4:92-100.

72. The World Health Organization (WHO). The Alcohol Use Disorders Identification Test: Guidelines for Use in Primary Care. Geneva: WHO; 2001.

73. Martinez P, Andia I, Emenyonu N, Hahn JA, Hauff E, Pepper L, et al. Alcohol use, depressive symptoms and the receipt of antiretroviral therapy in southwest Uganda. AIDS Behav. 2008;12(4):605-12.

74. Radloff LS. The CES-D Scale: a self-report depression scale for research in the general population. Appl Psychol Meas. 1977;1:385-401.

75. Kaharuza FM, Bunnell R, Moss S, Purcell DW, Bikaako-Kajura W, Wamai N, et al. Depression and CD4 Cell Count Among Persons with HIV Infection in Uganda. AIDS Behav. 2006;10(1):105-11.

76. Grinstead OA, Gregorich SE, Choi K-H, Coates T, The VHCTE Study Group. Positive and negative life events after counselling and testing: the Voluntary HIV-1 Counselling and Testing Efficacy Study. AIDS. 2001;15(8):1045-52.
77. Straus M. Measuring intra family conflict and violence: The Conflict Tactics (CT) Scales. J Marriage Fam. 1979;41(1):5-88.

78. Ellsberg M, Jansen HA, Heise L, Watts CH, Garcia-Moreno C. Intimate partner violence and women's physical and mental health in the WHO multicountry study on women's health and domestic violence: an observational study. Lancet. 2008;371(9619):1165-72.

79. Menzies NA, Berruti AA, Berzon R. The cost of providing comprehensive HIV treatment in PEPFAR-supported programs. AIDS. 2011;25(14):1753-60.

80. Kiene SM, Bateganya MH, Lule H, Wanyenze RK. The effect of motivational interviewing-based counseling during outpatient provider initiated HIV testing on high-risk sexual behavior in rural Uganda. AIDS Behav. 2016;20(9):1928-36.

81. McMahon JH, Elliot JH, Bertagnolio S, Kubiak R, Jordan MR. Viral suppression after 12 months of antiretroviral therapy in low- and middle-income countries: a systematic review. Bull World Health Organ. 2013;91(5):377-385E.

82. Barth RE, van der Meer JT, Hoepelman Al, Schrooders PA, van de Vijver DA, Geelen SP, et al. Effectiveness of highly active antiretroviral therapy administered by general practitioners in rural South Africa. Eur J Clin Microbiol Infect Dis. 2008;27(10):977-84.

83. Ndembi N, Goodall RL, Dunn DT, McCormick A, Burke A, Lyagoba F, et al. Viral rebound and emergence of drug resistance in the absence of viral load testing: a randomized comparison between zidovudine-lamivudine plus Nevirapine and zidovudine-lamivudine plus Abacavir. J Infect Dis. 2010; 201(1):106-13.

84. Landman R, Poupard M, Diallo M, Ngom Gueye NF, Diakhate N, Ndiaye B, et al. Tenofovir-emtricitabine-efavirenz in HIV-I-infected adults in Senegal: a 96-week pilot trial in treatment-naive patients. J Int Assoc Phys AIDS Care. 2009;8(6):379-84.

85. Samet JH, Freedberg KA, Stein MD, Lewis R, Savetsky J, Sullivan L, et al, Trillion virion delay: time from testing positive for HIV to presentation for primary care. Arch Intern Med. 1998;158(7):734-40.

86. Torian LV, Wiewel EW. Continuity of HIV-related medical care, New York City, 2005-2009: Do patients who initiate care stay in care? AIDS Patient Care STDs. 2011;25(2):79-88.

87. Seal PS, Jackson DA, Chamot E, Willig JH, Nevin CR, Allison JJ, et al. Temporal trends in presentation for outpatient HIV medical care 2000-2010: implications for short-term mortality. J Gen Intern Med. 2011;26(7):745-50.

\section{Submit your next manuscript to BioMed Central and we will help you at every step:}

- We accept pre-submission inquiries

- Our selector tool helps you to find the most relevant journal

- We provide round the clock customer support

- Convenient online submission

- Thorough peer review

- Inclusion in PubMed and all major indexing services

- Maximum visibility for your research

Submit your manuscript at www.biomedcentral.com/submit
Biomed Central 